\title{
CORTICOSTEROID INJECTIONS VERSUS PROLOTHERAPY FOR THE TREATMENT OF LATERAL EPICONDYLITIS: A RANDOMISED-CONTROLLED TRIAL
}

\author{
Utkarsh Pal' ${ }^{1}$ Nitin Kiradiya ${ }^{2}$
}

${ }^{1}$ Senior Resident, Department of Orthopaedics, Bundelkhand Medical College, Sagar.

${ }^{2}$ Assistant Professor, Department of Orthopaedics, Bundelkhand Medical College, Sagar.

\begin{tabular}{ll} 
ABSTRACT \\
\hline OBJECTIVE
\end{tabular}

To compare the efficacy of corticosteroid injection versus prolotherapy for the treatment of chronic lateral epicondylitis.

\section{DESIGN}

A prospective, randomised-controlled study.

\section{PARTICIPANTS}

Thirty-six subjects with clinically determined chronic (of duration three months or longer) lateral epicondylitis were recruited. All subjects noted pain intensity levels disturbing enough to prevent involvement in activities such as kneading dough, wringing clothes, lifting heavy weights and playing racquet sports.

\section{METHODS}

Subjects were randomised to get either corticosteroid injection or prolotherapy for treatment of chronic lateral epicondylitis. Each subject underwent injection at baseline followed by a second injection 3 weeks later.

\section{RESULTS}

Analysis in both the groups demonstrated statistically significant improvements in VAS and DASH scores within the prolotherapy group having significant changes recorded from baseline to 2 months and baseline to 6 months after initial treatment. The steroid group also recorded a clinically and statistically significant change for DASH only at both 2 month and 6 month followup. Analysis and comparison of the subjects completing the study gave no significant differences between the prolotherapy and the corticosteroid group for change in VAS or DASH, although at 1 year followup, 2 patients out of 20 patients reported sometimes pain in corticosteroid group while nobody reported pain in prolotherapy group. The study lacked sufficient power to draw conclusions from this finding. Aside from injection-associated pain, no other adverse reactions were documented.

\section{CONCLUSIONS}

Both corticosteroid and prolotherapy therapy were generally well tolerated and showed to provide benefit of short duration, however, prolotherapy gets preference on long term. Small sample size precludes determining whether one therapy is advantageous to the other. Larger controlled trials appear feasible and justified on the basis of these findings.

\section{KEYWORDS}

Prolotherapy, Lateral Epicondylitis, Injection Therapy, Tendinopathy.

HOW TO CITE THIS ARTICLE: Pal U, Kiradiya N. Corticosteroid injections versus prolotherapy for the treatment of lateral epicondylitis: a randomised-controlled trial. J. Evolution Med. Dent. Sci. 2016;5(72):5292-5295, D0I: 10.14260/jemds/2016/1199

\section{INTRODUCTION}

Lateral Epicondylosis (LE) ("tennis elbow") is a common condition around the elbow with an incidence of 4-7/1000 patients per year in primary care settings. ${ }^{1}$ Lateral Epicondylitis (LE) is a painful enthesopathy or tendinitis of the common extensor tendon at the lateral aspect of the elbow at the fibro-osseous junction. Histological specimens from patients with chronic LE suggest that tendinitis is not an acute inflammatory condition, but rather a disturbance in the normal tendon repair process associated with angiofibroblastic degeneration. Evidence argue that the cardinal factor involved is a degenerative rather than an inflammatory process. ${ }^{2}$

Financial or Other, Competing Interest: None.

Submission 29-07-2016, Peer Review 23-08-2016,

Acceptance 29-08-2016, Published 08-09-2016.

Corresponding Author:

Dr. Utkarsh Pal,

S/o. Mr. Laxman Singh Pal,

No. 9, Gayatri Nagar, Tansen Road

Gwalior-474002. Madhya Pradesh.

E-mail: utkarsh.pal@gmail.com

DOI: $10.14260 /$ jemds/2016/1199

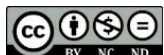

Numerous treatment options have been reported for LE. Conventional treatments include activity modification, rest, icing, nonsteroidal anti-inflammatory drugs, bracing and physical therapy. ${ }^{3}$ Nevertheless, none of these treatments has proved to be broadly effective. ${ }^{4}$ Perhaps, the most common treatment of LE is the corticosteroid injection, which is used to reduce inflammation in subjects with a variety of chronic tendinopathies. However, since histologic studies have not found inflammation as a prime attribute in this condition. The rationale for corticosteroid injection appears injudicious. There is some corroboration to suggest that inflammation may actually be a useful component of the response to inciting injury and the process of healing. Thus, inhibiting this process may actually be counterproductive. Several clinical trials have indicated limited efficacy for corticosteroid injections after 1 year. Given the lack of evidence for long-term satisfaction from corticosteroid injection, there appears to be a need for the investigation of other injectable agents. ${ }^{5}$

Injection protocols were formulated in the $1950 \mathrm{~s}$ by George Hackett, MD, a general surgeon in the US. ${ }^{6}$

Prolotherapy (PrT) is an injection-based treatment for chronic musculoskeletal pain. Sodium morrhuate (an extract 
of cod liver oil) and Dextrose (a form of glucose) are two common PrT injectants, which acts as an irritant for stimulation of wound healing and tissue repair into damaged ligaments and tendons by initiating an inflammatory cascade at the site of injection, which induces fibroblast proliferation and subsequent collagen synthesis thereby resulting in a tighter and stronger ligament or tendon. Therefore, as corticosteroid injections for chronic LE treatment are used to inhibit inflammation, the principal mode of action of prolotherapy is to instigate a very minimal inflammatory response to augment adequate healing and a more viable scar tissue formation, which results in stronger fibrous tissue that leads to decreased pain and improved function. ${ }^{7}$

Prolotherapy appears to be advantageous over corticosteroid injection. Prolotherapy may be preferable to patients as well as to doctor who are apprehensive about corticosteroid injection because of known adverse effects, such as tendon rupture, avascular necrosis, fat pad atrophy, skin depigmentation and also in hyperglycaemic and diabetic patients. Cost-effectiveness appears to be another major advantage of prolotherapy in the long-term treatment of chronic LE compared with corticosteroid injection because of its proposed ability to amend the disease process itself as opposed to addressing the symptoms. Prolotherapy could then lead to less additional healthcare-related disbursement such as repeated visits to clinics for a lengthy treatment course including repeat injections, physical therapy, analgesics as well as time taken from work. Therefore, we believe that this particular study is important.

\section{MATERIALS AND METHODS}

\section{Eligibility Criteria and Subject Selection}

Subjects with chronic LE who comes to outpatients were recruited.

Inclusion Criteria: Included, subjects between 18 and 60 years of age; a history of elbow pain over the area around lateral epicondyle varying in duration from 3 months to 2 years; pain on extension of wrist with resistance and/or middle finger test (extension of middle finger at the metacarpophalangeal joint with resistance); local tenderness to palpation at or just distal to the lateral epicondyle.

Exclusion Criteria: Includes, history of single or multiple corticosteroid injection within 6 months before enrolment for the study; another arm or forearm pathology, such as radial nerve compression; pregnancy; nursing; blood dyscrasias; history of nonspecific diffuse pain syndrome; history of inflammatory arthritis; workers; chronic regional pain syndrome; subjects with litigation pending/planned related to pain; hypersensitivity/contraindications to local anaesthetics or corticosteroids; depression; and history of narcotic abuse or a history of narcotic use for pain management for more than 1 month. Eligible patients were briefed about PrT and were invited to participate.

\section{Injection Methods}

Subjects qualifying for the study were randomised by the use 1:1 randomisation scheme of a random numbers table to receive any one of the two treatments. After through history and examination, subjects were randomised, obtained consent and were given injection at the first study visit. Both study medications were combined with a local anaesthetic solution (lidocaine $0.9 \%, \mathrm{NaCl}$ preservative-free $1 \%$ solution). All subjects received one of 2 study medications: (A) P2G (phenol $1.2 \%$, glycerine $12.5 \%$ and dextrose $12.5 \%$ in sterile water) with sodium morrhuate, a cod liver oil extract, a cocktail of irritant, osmotic and chemotactic agents; or (B) methylprednisolone acetate $40 \mathrm{mg} / \mathrm{mL}$. For the prolotherapy injection (protocol A), each $3 \mathrm{~mL}$ syringe was filled with $1.0 \mathrm{~mL}$ of Xylocaine $1 \%, 0.9 \mathrm{~mL}$ of phenol, glycerine and d-glucose (P2G) and $0.1 \mathrm{~mL}$ of sodium morrhuate. For the corticosteroid group (protocol B), each $3 \mathrm{~mL}$ syringe was filled with $1.0 \mathrm{~mL}$ of Xylocaine $1 \%$ and $1.0 \mathrm{~mL}$ of methylprednisolone. A 25-gauge was used for all injections. Both the solutions had a similar milky white appearance. Then, using standard injection procedure for lateral epicondylitis injection was given. Subjects were monitored for half an hour for an allergic reaction after injection. Each subject was given a 48-hour prescription for diclofenac whenever required.

\section{Outcome Measures}

For outcome measures for all subjects were assessed at baseline 3, 8, 16 and 52 weeks. The primary outcome measure tool was the Visual Analogue Scale (VAS) and for assessment secondary outcome tool used was the Disabilities of the Arm, Shoulder and Hand (DASH) questionnaire. The VAS is a widely used and clinically validated scale of pain severity with good sensitivity to change in pain symptoms. In this study, a 100mm vertical line labelled with "no pain" on the bottom and "pain as bad as it could be" on the top was used to answer the question: "How would you rate your elbow pain?". DASH is intended to measure how much difficulty a subject has when performing common functional tasks and activities. The DASH is composed of a 30-item questionnaire with 5 response options for each item with a scale ranging from 0 , which indicates "least disability," to 100 , which indicates "most disability." It incorporates questions related to functional constraints, symptoms and psychosocial issues. Other data collected at baseline included age, gender, side of involvement and time since onset of symptoms.

At the 3-weeks followup, the subject underwent repeat physical examination and assessment including VAS, DASH. All subjects underwent repeat injection with either a prolotherapy or corticosteroid solution on the basis of their initial assignment. Subjects were reassessed again at 8 weeks and six months after study entry at which time the VAS and DASH were repeated. No further injections were performed. At 52 weeks, subjects were asked (by telephone) three followup questions: how much elbow pain they had (none, mild, moderate or severe), whether elbow pain affected their ability to perform activities of daily living and whether they used alternate therapies for pain after the injections.

\section{Statistical Analysis}

A difference of 02 for VAS and 12 for DASH was considered clinically significant. ${ }^{8,9} \mathrm{An}$ inadequate number of patients were engaged in our study and consequently a direct comparison of the 2 injection techniques could not be statistically assessed. To analyse the change in scores for both VAS and DASH between the 2 groups, an exploratory analysis was performed.

Differences between the two groups were then assessed with t-test (paired or independent samples for within and in- 
between groups comparisons, respectively). Within each study group, analysis was performed by the use of paired t-tests to establish, which group showed improvement from baseline to the 3-month followup. A P-value of 0.05 was considered statistically significant. The same procedure was used to determine change in VAS and DASH from baseline to the 6month followup as a secondary endpoint.

\section{RESULTS}

Despite the initial goal to enrol 60 subjects to complete this study, only 40 subjects were actually enrolled of whom 36 completed the study. Enrolment was stopped because of lack of funding. Of the 40 patients who enrolled, one subject was dropped out from the study before receiving treatment after onset of symptoms consistent with bicipital tendinitis thus complicating interpretation. One subject got lost in followup and another one received one treatment each of both prolotherapy and corticosteroids and was thus excluded from further analysis. One participant withdrew from the prolotherapy group because of excessive pain; no other adverse reactions were reported. Therefore, analysis was performed with the use of data from the 36 patients who completed the study. Luckily, we have got 18 subjects in each group. Subjects' ages ranged from 19 to 50 years with a mean age of 35.7. Duration of elbow pain ranged from 0.5 years to 4 years. Baseline demographics, pain and function scores were comparable between the two groups. Eleven subjects who completed the study were women. Patients ranged in age from 35 to 50 years. Symptoms of LE were present for more than 3 months in all participants. All patients registered were working as their work required and had attained an educational background at higher secondary level or greater. Within each study group, analyses disclosed reduction in pain as measured by the VAS as well as DASH group from baseline to 3 months and 6 months. No significant differences in the amount of change were observed between the prolotherapy and corticosteroid groups at 3 or 6 months with any of the 2 scales. At the 52-week followup, all subjects of prolotherapy group reported no elbow pain while in corticosteroid group $11.1 \%(n=2)$ reported mild pain. Activities of daily living were unaffected in both groups. None of the subjects reported using or seeking additional therapy. Sixteen out of eighteen study participants receiving steroid therapy and all participants receiving prolotherapy reported overall satisfaction with the outcome achieved upon completion of the study at one year. Side effects of injection therapy were minimal. Most of the subjects experienced, expected, self-limited post-injection pain. These symptoms resolved with ibuprofen.

\section{DISCUSSION}

This Randomised Controlled Trial (RCT) reports improvements within each of the 2 treatment groups over the course of the study suggesting some degree of efficacy for both treatments. However, at the end of one year, two participants of corticosteroid group started experiencing mild heaviness in extensor muscle bulk along with sometimes pain in lateral epicondyle. This finding could suggest longer efficacy duration for prolotherapy.

Improved outcomes in the current study may occur from effects hypothesised in the literature. First, concentrated dextrose and sodium morrhuate have sclerosant qualities. 10 When used to destroy neovessels in a procedure very similar to prolotherapy, the sclerosant polidocanol has been reported to reduce pain in lateral epicondyle.11,12 Achilles tendinopathies ${ }^{13}$ and patellar tendinopathy ${ }^{14}$ and injections with dextrose and sodium morrhuate may have sclerosed neovessels and associated new nerves though we did not attempt to visualise neovascularity using ultrasound. Second, bleeding from needle trauma and tissue expansion in the potential space adjacent to tendon insertions may mimic the effect of fenestration and autologous blood injections reported in the Connell and Mishra studies. Finally, dextrose and sodium morrhuate have been shown to affect the strength and size of stretch-injured ligaments in an animal model perhaps by an inflammatory mechanism. ${ }^{15}$ Given that tendinopathies at various anatomical sites likely share pathophysiological mechanisms, prolotherapy may be relevant treatment for tendinopathy other than LE. However, studies says at high concentrations, sodium morrhuate in vitro is toxic to granulocytes, red blood cells and endothelial cells. ${ }^{16}$

In a recent double-blind randomised controlled trial, Scarpone et al examined the effect of prolotherapy on resting elbow pain, extension and grip strength in 24 adults with at least 6 months of refractory LE. ${ }^{17}$ Results revealed that, compared with control subjects, those who received prolotherapy reported improved pain scores at each of the 3 reporting intervals. This finding was determined to be significant when compared with baseline. This effect was maintained at long-term followup. In our present RCT, subjects also reported improvement in pain as compared with baseline with no report of any adverse events.

Prolotherapy has been proposed as a potential therapy for chronic LE and is used by some physicians to treat this condition and other enthesopathies. Prolotherapy is believed to produce a controlled inflammatory response and to stimulate adequate fibroblastic proliferation and connective tissue repair. ${ }^{18}$ This result has been shown in multiple studies in which the authors used different proliferants. For example, biopsy samples examined after use of proliferant containing $2.5 \%$ phenol, $25 \%$ dextrose and 25\% glycerin (P2G) demonstrated a transient inflammatory reaction sufficient to create a fibroblast response.

These studies, in which the investigators have used a variety of proliferant solutions and animal models versus human subjects, illustrate the basic mechanism of prolotherapy, in which injection of an irritant solution triggers a localised inflammatory cascade similar to that which occurs in the normal healing process whereby fibroblastic hyperplasia and collagen formation lead to tightening, thickening and strengthening of the ligaments or tendons. This, in turn, results in stronger connective tissue with improved biomechanics, joint function and decreased pain. ${ }^{19}$ To date, no specific prolotherapy guidelines exist and published clinical studies show significant differences in treatment protocols with little attempt at standardisation. In addition to the use of different proliferant solutions, studies vary in the number of treatments given, treatment intervals, proliferant doses and concentrations and adjunct therapies used. ${ }^{20}$ Three solutions are commonly used in proliferant therapy including D-glucose (Dextrose), phenol-glucose glycerin (P2G), sodium morrhuate or combinations of these (Dextrose and sodium morrhuate, in addition to Mepivacaine and cyanocobalamin, known as Pomeroy solution). ${ }^{20}$ 


\section{LIMITATIONS}

There are various constraints of the study excluding the four randomised dropouts in the outcome analysis could have introduced bias. But, there are situations in which excluding drop-out is appropriate. In the current study, this was justified because the loss to follow up was balanced in each group. The major weakness of our study was a small sample size thereby limiting conclusions that can be drawn from the data. The study was underpowered and not able to detect statistical significance between groups. Future studies may consider using multiple centres or less stringent inclusion criteria. Another limitation of the study was the limited number of injections given. Only two injections of prolotherapy were given because this study allowed a direct comparison of prolotherapy to the established corticosteroid injection treatment. Although, there is no established guidelines with respect to the secure number of injections or a guided interval between injections. In our musculoskeletal medicine practice, we try to limit corticosteroid injections to 3-4 per year. ${ }^{13}$ Therefore, it was thought that having subjects to undergo only 2 injections may as an effort to limit adverse effects of steroids. Standard prolotherapy treatment consists of more than 2 injection sets when symptoms are not clinically relieving. However, because a standard of care practice regarding the number of corticosteroid injections does not currently established. The issue is raised here on the feasibility of conducting a study using more injection sets. One-year followup questions were non-standard and while clinically relevant were difficult to relate to the quantitative data. Strength of the studies include randomised controlled design, multiple standard, minimal missing data and a large consistent effect size for pain both group.

\section{CONCLUSION}

To our knowledge, this is the one of the few RCT comparing prolotherapy with corticosteroid injection for the treatment of chronic LE and thereby assessing the use of prolotherapy for this patient population. Prolotherapy with dextrose and sodium morrhuate was well tolerated and improved pain compared to corticosteroid injections. Our results propose that prolotherapy may be a helpful alternative to corticosteroid injection and may provide a basis for undertaking larger, more definitive studies. Prolotherapy performed by a trained operator is a reasonable therapeutic option for patients with refractory chronic LE, which is a safe, economical and effective intervention. Rural physicians should consider it as a treatment option for lateral epicondylosis, especially when the other conservative approaches have failed to treat the condition.

\section{REFERENCES}

1. Hamilton PG. The prevalence of humeral epicondylitis: a survey in general practice. J R Coll Gen Pract 1986;36(291):464-5.

2. Labelle H, Fallaha M, Newman N, et al. The treatment of tennis elbow: a critical review of the literature. J Bone Joint Surg Br 1990;72:536.

3. Labelle H, Guibert R, Joncas J, et al. Lack of scientific evidence for the treatment of lateral epicondylitis of the elbow. An attempted meta-analysis. J Bone Joint Surg Br 1992;74(5):646-51.
4. Kivi P. The etiology and conservative treatment of lateral epicondylitis. Scand J Rehabil Med 1983;15(1):37-41.

5. Ollivierre CO, Nirschl RP. Tennis elbow current concepts of treatment and rehabilitation. Sports Med 1996;22(2):133-9.

6. Hackett GS, Hemwall GA, Montgomery GA. Ligament and tendon relaxation treated by prolotherapy. $5^{\text {th }}$ ed. Oak Park: Gustav A. Hemwall 1993.

7. Carayannopoulos A, Borg-Stein J, Sokolof J, et al. Prolotherapy versus corticosteroid injections for the treatment of lateral epicondylosis: a randomized controlled trial. PMR 2011;3(8):706-15.

8. Farrar JT, Young JP, LaMoreaux L, et al. Clinical importance of changes in chronic pain intensity measured on an 11point numerical rating scale. Pain 2001;94(2):149-58.

9. Beaton DE, Katz JN, Fossel AH, et al. Measuring the whole or the parts? Validity, reliability, and responsiveness of the disabilities of the arm, shoulder, and hand outcome measure in different regions of the upper extremity. J Hand Ther 2001;14(2):128-46.

10. Bartholomew JR, King T, Sahgal A, et al. Varicose veins: newer better treatments available. Clev Clin J Med 2005;72(4):312-28.

11. Zeisig E, Ohberg L, Alfredson H. Extensor origin vascularity related to pain in patients with tennis elbow. Knee Surg Sports Traumatol Arthrosc 2006;14(7):659-63.

12. Zeisig E, Ohberg L, Alfredson H. Sclerosing polidocanol injections in chronic painful elbow-promising results in a pilot study. Knee Surg Sports Traumatol Arthrosc 2006;14(11):1218-24.

13. Alfredson $\mathrm{H}$, Ohberg L. Sclerosing injections to areas of neovascularization reduce pain in chronic achilles tendinopathy: a double-blind randomised trial. Knee Surg Sports Traumatol Arthrosc 2005;13(4):338-44.

14. Hoksrud A, Ohberg L, Alfredson $\mathrm{H}$, et al. Ultrasoundguided sclerosis of neovessels in painful chronic patellar tendinopathy. Am J Sports Med 2006;34(11):1738-46.

15. Aneja A, Karas SG, Weinhold PS, et al. Suture plication, thermal shrinkage and sclerosing agents. American Journal of Sports Medicine 2005;33(11):1729-34.

16. Stroncek DF, Hutton SW, Silvis SE, et al. Sodium morrhuate stimulates granulocytes and damages erythrocytes and endothelial cells: probable mechanism of an adverse reaction during sclerotherapy. J Lab Clin Med 1985;106(5):498-504.

17. Scarpone M, Rabago D, Arbogest G, et al. The efficacy of prolotherapy for lateral epicondylosis: a pilot study. Clin J Sports Med 2008;18(3):248-54.

18. Banks AR. A rationale for prolotherapy. J Orthop Med 1991;13(3):54-9.

19. Linetsky FS, Miguel R, Saberski L. Pain management with regenerative injection therapy (RIT). In: Weiner RS, edr. Pain management: a practical guide for clinicians. Washington, DC: CRC Press 2002:381-402.

20. Haslock I, Macfarlane D, Speed C. Intra-articular and soft tissue injections: a survey of current practice. $\mathrm{Br} \mathrm{J}$ Rheum 1995;34(5):449-52. 